\title{
Recurrent Painful Ophthalmoplegic Neuropathy Affecting Right Oculomotor Nerve in 10-Year-Old Male. Case Report
}

Sharmila Segar,' Chandni Duphare,' Osemelu Aburime.

\begin{abstract}
Background: Recurrent painful ophthalmoplegic neuropathy (RPON), formerly known as ophthalmoplegic migraine (OM), is a poorly understood condition that presents with recurrent unilateral headaches and at least one ocular cranial nerve (CN) palsy, generally in childhood. There has been ongoing debate about whether the etiology of this disorder is neuropathic or related to migraines. The Case: We present a case about a 10-year-old male with his third presentation of RPON, repeatedly affecting his right oculomotor nerve. His treatment choices are discussed, along with associated outcomes. The patient was treated with topiramate with resolution of his symptoms occurred within one month. Conclusion: As the annual incidence of RPON is rare at fewer than 1 case per million people, clear documentation of observed cases with treatment failures and successes is key to building evidence for future management.
\end{abstract}

Key Words: Ophthalmoplegic Migraine; Cranial Nerve Diseases; Oculomotor Nerve (Source: MeSH-NLM).

\section{Introduction}

Recurrent painful ophthalmoplegic neuropathy (RPON) is a condition that can present with days to weeks of ocular cranial nerve palsies and debilitating headaches. The majority of cases of RPON are found in patients with a history of migraines, with the associated headache having migrainous features, including photophobia, phonophobia, nausea, and vomiting. ${ }^{1}$ A headache lasting several days to a week is typically the first sign of RPON, with high intensity in the periorbital and/or retro-orbital region. Ophthalmoplegia tends to present during or after the headache. Based on 84 cases, median age of first attack is 8 years, with a range of 7 months to 50 years, while most cases start in childhood. ${ }^{2}$ The condition is very rare, with a reported annual incidence of 0.7 per million based on a population of 615,000 studied over 10 years. ${ }^{3}$ While this condition is rare, further understanding of the condition and treatment is warranted, as associated headaches and ocular impairments can result in lost productivity from missed school or work days, along with uncertainty about recurrence.

We encountered a pediatric patient with a history of migraines during his third case of RPON. He was treated with migraine prophylactic medications with an increase in his dose of topiramate and the continuation of his regularly scheduled dose of verapamil. The treatment of RPON is not standardized, and this case illustrates that the use of migraine medications without steroids may be efficacious. The pathophysiology of RPON is discussed below, with proposed etiologies including migrainous, neuropathic, and vasculopathic.

\section{The Case}

A 10-year-old Hispanic male presented with a history of right-sided frontal headache without aura lasting for 4 days. The patient reported vomiting and phonophobia on days $1-2$. During days $3-4$, the patient developed diplopia and ptosis of the right eye (OD). At the time of the clinic visit, the headache had subsided but the patient was still unable to open OD. He denied any eye pain, injection, discharge, blurry vision, numbness, or weakness. He denied fever, upper respiratory illness, sick contacts, recent vaccinations, or trauma.

\section{Key Points: \\ - Recurrent painful ophthalmoplegic migraine is a condition defined as having 1) unilateral headache with ipsilateral paresis of at least 1 ocular cranial nerve; 2) at least two attacks; 3 ) exclusion of orbital, parasellar, or posterior fossa lesions; and 4) lack of more fitting headache diagnosis. \\ - While pathophysiology and treatment of RPON are unclear, steroids and migraine prophylaxis medications may improve symptoms. - Larger scale studies are essential in determining which treatment regimens most definitively improve outcomes.}

The patient was diagnosed with migraines at age 5 and had 2-3 severe migraine episodes per year. When he was 8 years old, he had his first presentation of ptosis OD following headache, which resolved after several weeks. When he was 9 , he was hospitalized for one night for an episode of headache that lasted 4 days as well as ptosis OD and horizontal diplopia. He underwent appropriate investigations and was started on migraine prophylaxis. The patient was discharged with verapamil titrated up to $40 \mathrm{mg}$ BID for 4 weeks and prednisone for 6 days. After his previous attacks, his current medications were topiramate (25 mg PO BID) and verapamil ( $40 \mathrm{mg}$ PO daily). It's worth noting that the patient's family history did not include oculomotor palsy.

On ocular exam, visual acuity was symmetric at $20 / 25$ in both eyes (OU). Extraocular movements (Figure 1) showed right eye exotropia in primary gaze, -4 adduction, -4 elevation, -3 depression and normal abduction. The left eye (OS) had full extraocular movements. The remainder of the ocular examination was within normal limits.

Complete blood count (CBC), comprehensive metabolic panel (CMP), erythrocyte sedimentation rate (ESR), C-reactive protein (CRP), angiotensin converting enzyme (ACE), hemoglobin ArC ( $\mathrm{BbA}>\mathrm{C}$ ), rheumatoid factor (RF), antinuclear antibody (ANA), lysozyme, and Quantiferon Cold were within normal limits. Lumbar puncture CSF studies were unremarkable, reducing suspicion for treatable infections, inflammatory or neoplastic etiologies.

${ }^{1}$ Medical College of Georgia, Augusta University, Augusta, Georgia, USA.

About the Author: Sharmila Segar is a fourth-year medical student at the Medical College of Georgia, pursuing a career in ophthalmology. 


\section{Case Report}

Figure 1. Extraocular Movements with Oculomotor Nerve Palsy.
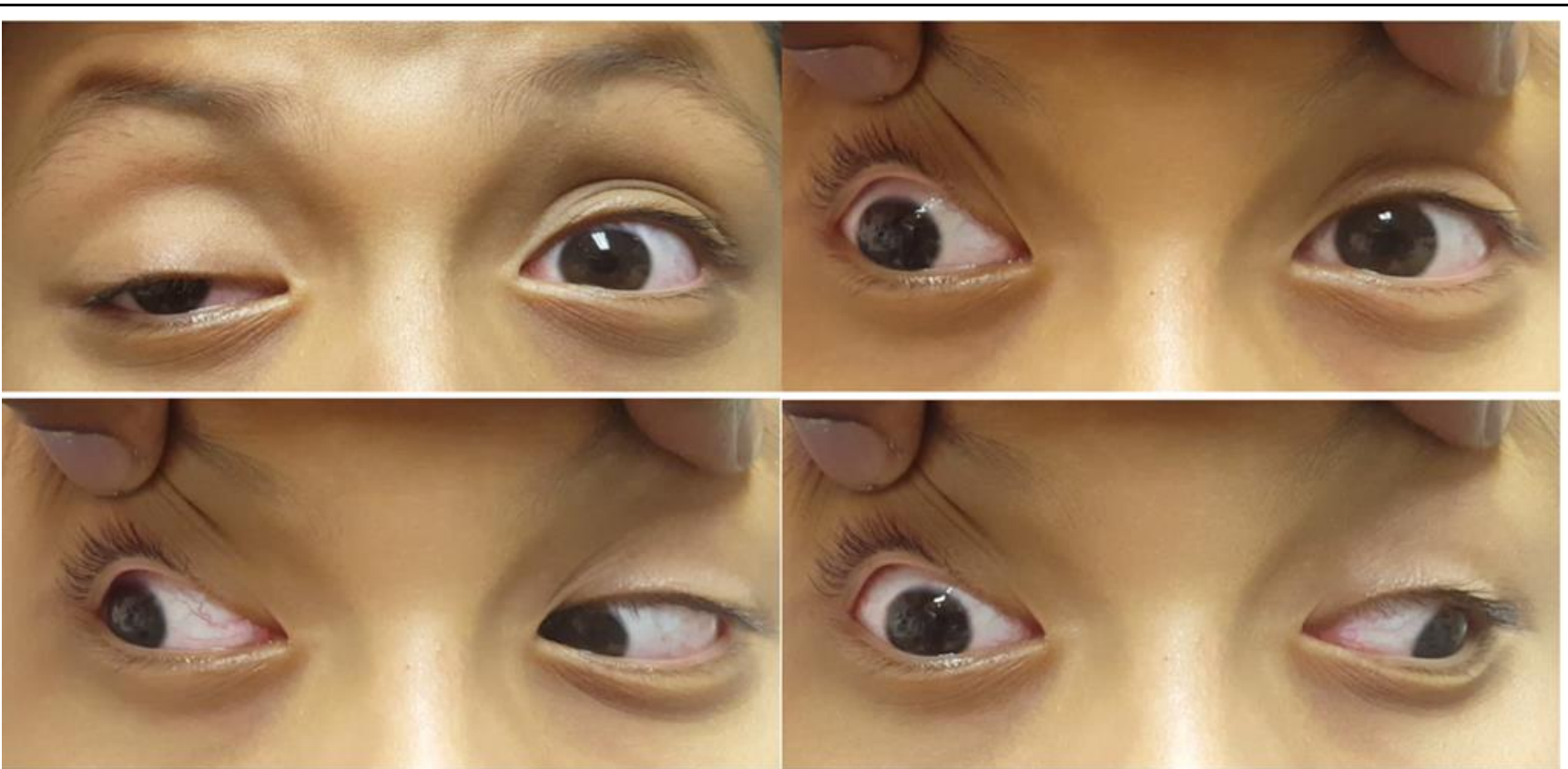

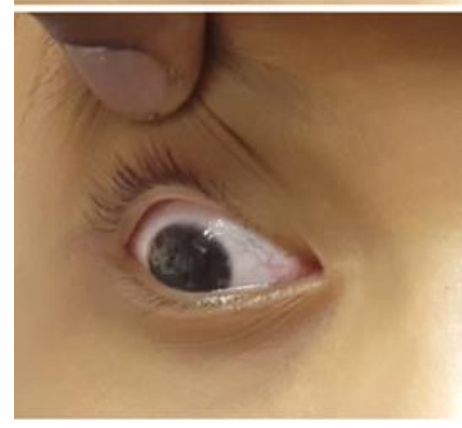

Magnetic resonance imaging (MRI) of the brain with contrast (Figure 2) showed mild loss of volume and abnormal enhancement with the contrast of the cisternal portion of the right oculomotor nerve. The amount of abnormal enhancement and the degree of loss of volume appeared slightly decreased in comparison to the prior MRI from age 9. otherwise, there were no changes in comparison to the prior MRI.

The patient was treated by increasing the dose of topiramate from 50 to $75 \mathrm{mg}$ BID. The patient's parents were informed to contact the eye clinic if the condition worsened in order for steroids to be prescribed. One month after the current presentation, the patient had near full resolution of ptosis and impaired extraocular movements.

During the episode that resulted in hospitalization at age 9, patient was discharged with verapamil titrated up to $40 \mathrm{mg}$ twice daily with a fourweek supply, and a six-day prednisone course. The patient's mother reported that verapamil did not decrease the intensity of his headache, and that the ptosis did not resolve with the completion of prednisone. All three of the patient's ophthalmoplegic episodes resolved in approximately one month. He is being followed by pediatric neurology and ophthalmology.

\section{Discussion}

The name change from "ophthalmoplegic migraine" to "recurrent painful ophthalmoplegic neuropathy" highlights how the condition was previously thought to be a migraine variant, while it is now predominantly thought to arise from a recurrent demyelinating neuropathy. The change was first reported by the International Classification of Headache Disorders in its 3 rd edition (published in

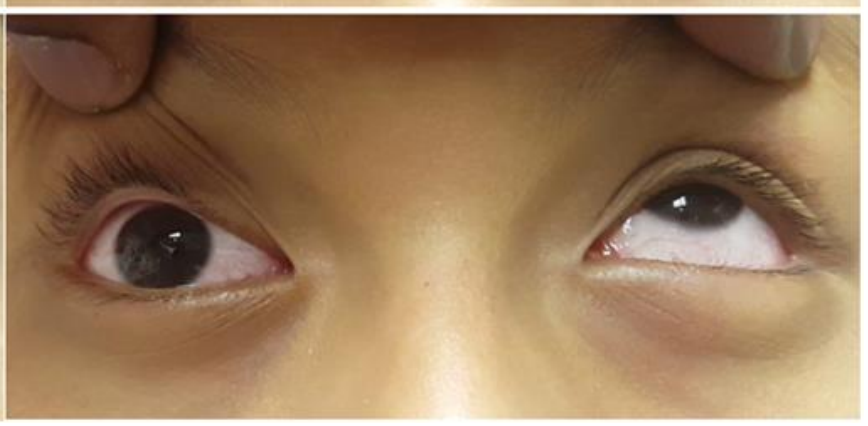

2013). The four diagnostic criteria include: 1) unilateral headache with ipsilateral paresis of at least 1 ocular cranial nerve; 2) having at least two attacks; 3) exclusion of orbital, parasellar, or posterior fossa lesions; and 4) lack of a more fitting headache diagnosis. ${ }^{4}$ The third cranial nerve is most commonly involved.

\section{Pathophysiology}

The pathophysiology of the RPON remains uncertain. Regarding a migraine-related etiology, swelling of the internal carotid or posterior cerebral artery vessel walls could lead to occlusion of branches supplying the cisternal portion of the oculomotor nerves, leading to vasogenic edema contributing to ophthalmoplegia and abnormal contrast enhancement on MRI. ${ }^{1}$ Neuropeptides involved in migraines, including calcitonin-gene-related peptide may also contribute to inflammation at the blood brain barrier. ${ }^{1,5}$

MRI findings associated with RPON are typically not present with migraines. The pain associated with migraines tend not to last for longer than 72 hours. Cranial nerve palsies can be associated with pain lasting for multiple days or weeks. ${ }^{6}$ In a study of motor palsies, some were associated with diabetic neuropathy, with one-third of patients having pain preceding visual symptoms. ${ }^{6}$

It has also been proposed that RPON is the result of a vasculitis-type immunologic response in the nerve or a post-viral inflammatory condition.? In 2018, Huang C et al. suggested that a multifactorial etiology, including neuropathic and migrainous, should be maintained given the existing evidence. ${ }^{8}$ Some even propose a microvascular, ischemic etiology to the disease. 9 In cases of patients with RPON, both 
Figure 2. MRI with Contrast Showing Mild Loss of Volume and Abnormal Enhancement of the Cisternal Portion of the Right Oculomotor Nerve.

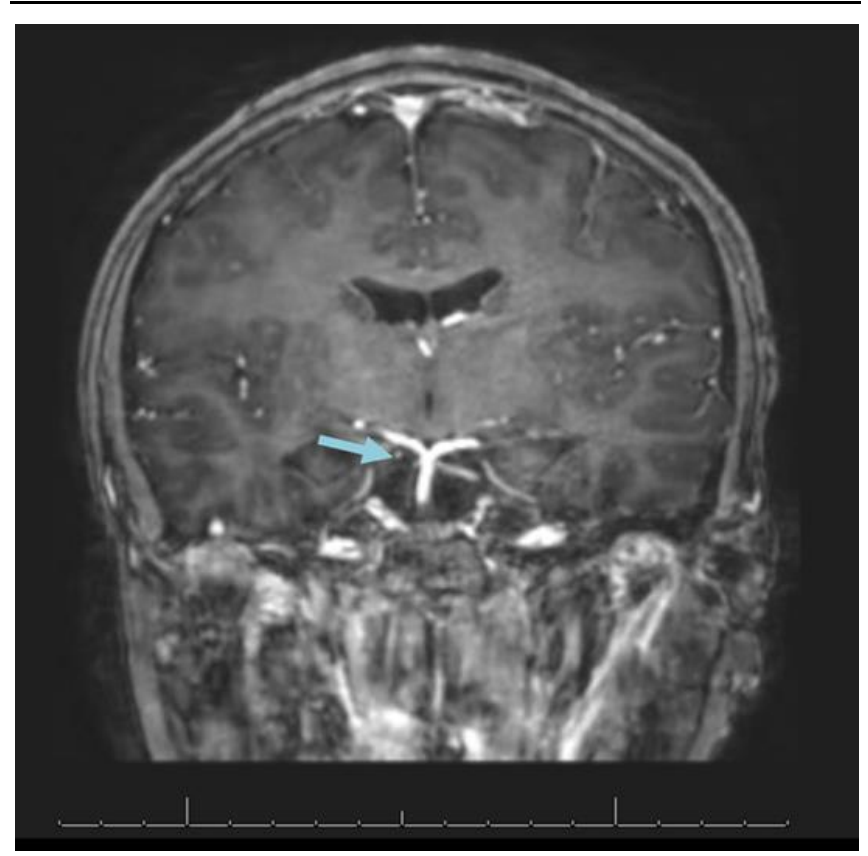

neuromuscular hamartoma and schwannoma have been found originating from the oculomotor nerve, which have been discussed as being primary pathologic factors or even mimickers of RPON. ${ }^{10-13}$ Further research and evaluation of autopsy cases are required in order to understand how this condition arises.

\section{Diagnosis}

In order to differentiate between RPON and infectious, noninfectious inflammatory conditions, and malignancies, there is a need for clinical evaluation, blood tests, lumbar puncture, and imaging studies. ${ }^{14}$ The first attack is generally incorrectly considered to be aneurysm, trauma, infection, or recent immunization, with no recurrent attack present to be included in diagnostic criteria of RPON. ${ }^{15}$ Lab tests can include CBC, CMP, ESR, CRP, ACE, HbA1C, RF, ANA, lysozyme, human leukocyte antigen B27 (HLA-B27), and antineutrophil cytoplasmic antibody (ANCA). These tests tend to be negative in RPON. ${ }^{2}$

MRI with gadolinium contrast during both of the patient's episodes at ages 9 and 10 indicated abnormal enhancement and loss of volume of the cisternal portion of the right oculomotor nerve. The abnormal enhancement and degree of volume loss were similar in comparison to the first MRI. A study showed that contrast enhancement of the cisternal segment of the affected oculomotor nerve is a common finding during the acute phase of oculomotor RPON, which was found in all six participants. ${ }^{14}$ Focal thickening at the exit of the nerve in the interpeduncular cistern was present in most patients, and whole nerve thickening was present in one patient. This enhancement was later found to be almost fully resolved 7 to 9 weeks later. A lack of significant MRI findings should not exclude RPON. ${ }^{16}$ In our case, instead of focal or whole nerve thickening, the oculomotor nerve had a mild asymmetric loss of volume.

\section{Treatment}

Treatment for RPON is not clearly established. In one study of 26 patients, steroid use improved symptoms in over half of patients, with unclear benefit to the other patients with RPON. ${ }^{2}$ One case reported resolution of painful ophthalmoplegia within 7 days of initiation of oral prednisone $2 \mathrm{mg} / \mathrm{kg} /$ day, followed by steroid taper and initiation of migraine prophylaxis. ${ }^{15}$ These mixed results with steroids have been documented, with optimal prophylactic and acute medications not clearly established. ${ }^{17}$ In a study with two steroid responsive patients, cyproheptadine hydrochloride was effectively used to prevent RPON by reducing vascular edema around the affected nerve. ${ }^{18}$

Medications used for migraine prophylaxis have not been well studied for use in RPON. In this case, the patient's first ophthalmoplegic event at 8 years was defined by ptosis OD with headache, resolving without any medication. At age 9, his second event presented with diplopia, ptosis OD, and headache, and he was prescribed six-day prednisone course as well as $40 \mathrm{mg}$ verapamil BID for 4 weeks. His mother reported unclear benefit. For his third presentation at age 10, his regular dose of topiramate was increased from 50 to $75 \mathrm{mg}$ BID. It is unclear whether his resolution occurring consistently at 1 month after initial presentation was because of the medications or the natural course of the disease.

\section{Prognosis}

While there is still great potential for further understanding of pathophysiology and treatment of RPON, the prognosis is excellent. Some deficits may persist after multiple attacks while this occurrence is unlikely after one attack. ${ }^{17,19}$ In order to prevent events of RPON and long-term sequelae, further research is warranted.

\section{Conclusion}

RPON is a disease with an unclear pathophysiology and many proposed treatments. With a better understanding of the pathophysiology, the most efficacious treatment regimens may be elucidated. Considering the rare nature of the disease, documentation of cases of RPON is essential. Prospective research goals should include randomized clinical trials for specific therapeutic agents.

\section{Consent}

Written informed consent was obtained from the patient for publication of this case report and any accompanying images. A copy of the written consent is available for review by the Editor-in-Chief of this journal. 


\section{Case Report}

\section{References}

1. Forderreuther $S$, Ruscheweyh R. From ophthalmoplegic migraine to cranial neuropathy. Curr Pain Headache Rep. 2015 Jun;19(6):21.

2. Celfand AA, Gelfand JM, Prabakhar P, Coadsby PJ. Ophthalmoplegic "migraine" or recurrent ophthalmoplegic cranial neuropathy: new cases and a systematic review. J Child Neurol. 2012 Jun;27(6):759-66.

3. Hansen SL, Borelli-Moller L, Strange P, Nielsen BM, Olesen J. Ophthalmoplegic migraine: diagnostic criteria, incidence of hospitalization and possible etiology. Acta Neurol Scand. 1990 Jan;81(1):54-60.

4. Headache Classification C, Olesen J, Bousser MC, Diener HC, Dodick D, First M, et al. New appendix criteria open for a broader concept of chronic migraine. Cephalalgia. 2006 Jun;26(6):742-6.

5. Carlow TJ. Oculomotor ophthalmoplegic migraine: is it really migraine? I Neuroophthalmol. 2002 Sep;22(3):215-21.

6. Wilker SC, Rucker JC, Newman NJ, Biousse V, Tomsak RL. Pain in ischaemic ocular motor cranial nerve palsies. Br J Ophthalmol. 2009 Dec;93(12):1657-9.

7. Lal V. Ophthalmoplegic migraine: past, present and future. Neurol India. 2010 JanFeb;58(1):15-9.

8. Huang C, Amasanti M, Lovell B, Young T. Recurrent painful ophthalmoplegic neuropathy. Pract Neurol. 2017 Aug;17(4):318-20.

9. Manzouri B, Sainani A, Plant C, Lee J, Sloper J. The aetiology and management of long-lasting sixth nerve palsy in ophthalmoplegic migraine. Cephalalgia. 2007 $\operatorname{Mar} ; 27(3): 275-8$.

10. Akimoto J, Fukami S, Hashimoto R, Haraoka J. Neuromuscular hamartoma is a possible primary pathology of oculomotor ophthalmoplegic migraine. Cephalalgia. 2012 Jan;32(2):171-4.
11. Kawasaki A. Oculomotor nerve schwannoma associated with ophthalmoplegic migraine. Am J Ophthalmol. 1999 Nov;128(5):658-60

12. Bisdorff AR, Wildanger G. Oculomotor nerve schwannoma mimicking ophthalmoplegic migraine. Cephalalgia. 2006 Sep;26(9):1157-9.

13. Shin RK, Mejico LJ, Kawasaki A, Purvin VA, Moster ML, Younge BR, et al. Transien ocular motor nerve palsies associated with presumed cranial nerve schwannomas. I Neuroophthalmol. 2015 Jun;35(2):139-43.

14. Mark AS, Casselman J, Brown D, Sanchez J, Kolsky M, Larsen TC, 3rd, et al. Ophthalmoplegic migraine: reversible enhancement and thickening of the cisterna segment of the oculomotor nerve on contrast-enhanced MR images. AJNR Am Neuroradiol. 1998 Nov-Dec;19(10):1887-91.

15. Roy M, Chosh J, Deb S, Pandit N. Childhood steroid-responsive ophthalmoplegic migraine. J Pediatr Neurosci. 2011 Jan;6(1):69-71.

16. Prats JM, Mateos B, Garaizar C. Resolution of MRI abnormalities of the oculomoto nerve in childhood ophthalmoplegic migraine. Cephalalgia. 1999 Sep;19(7):655-9

17. Levin M, Ward TN. Ophthalmoplegic migraine. Curr Pain Headache Rep. 2004 Aug;8(4):306-9.

18. Sugiyama N, Hamano S, Tanaka M, Mochizuki M, Nara T. [MRI findings and effectiveness of cyproheptadine in two patients with ophthalmoplegic migraine]. No To Hattatsu. $2002 \mathrm{Nov} ; 34(6): 533-7$. Japanese

19. Crevits L, Verschelde H, Casselman J. Ophthalmoplegic migraine: an unresolved problem. Cephalalgia. 2006 0ct;26(10):1255-9.

\section{Acknowledgments}

We would like to thank the Medical College of Georgia Department of Ophthalmology for their help in promoting medical student exposure to new cases. Conflict of Interest Statement at Funding

The Authors have no funding, financial relationships or conflicts of interest to disclose.

\section{Author Contributions}

Conceptualization: SS, and OS. Validation: OS. Formal Analysis: SS, and CD. Data Curation: SS, and OS. Resources: SS, and CD. Writing - Original Draft: SS. Writing - Review A Editing: SS, and CD. Supervision: OS

Cite as:

Segar S, Duphare C, Aburime 0. Recurrent Painful Ophthalmoplegic Neuropathy Affecting Right Oculomotor Nerve in 10-Year-Old Male. Case Report. Int Med Students. 2018 Sep-Dec;6(3):114-7.

This work is licensed under a Creative Commons Attribution 4.0 International License 